\title{
The heterodimeric structure of heterogeneous nuclear ribonucleoprotein C1/C2 dictates 1,25-dihydroxyvitamin D-directed transcriptional events in osteoblasts
}

\author{
Thomas S Lisse ${ }^{1,2}$, Kanagasabai Vadivel ${ }^{1}$, S Paul Bajaj ${ }^{1}$, Rui Zhou ${ }^{1}$, Rene F Chun ${ }^{1}$, Martin Hewison ${ }^{1}$ and John S Adams ${ }^{1}$
}

Heterogeneous nuclear ribonucleoprotein (hnRNP) C plays a key role in RNA processing but also exerts a dominant negative effect on responses to 1,25-dihydroxyvitamin $\mathrm{D}\left(1,25(\mathrm{OH})_{2} \mathrm{D}\right)$ by functioning as a vitamin $\mathrm{D}$ response element-binding protein (VDRE-BP). hnRNPC acts a tetramer of hnRNPC1 (huC1) and hnRNPC2 (huC2), and organization of these subunits is critical to in vivo nucleic acid-binding. Overexpression of either huC1 or huC2 in human osteoblasts is sufficient to confer VDRE-BP suppression of $1,25(\mathrm{OH})_{2} \mathrm{D}$-mediated transcription. However, huC1 or huC2 alone did not suppress $1,25(\mathrm{OH})_{2} \mathrm{D}$-induced transcription in mouse osteoblastic cells. By contrast, overexpression of huC1 and huC2 in combination or transfection with a bone-specific polycistronic vector using a "self-cleaving" $2 \mathrm{~A}$ peptide to co-express huC1/C2 suppressed 1,25D-mediated induction of osteoblast target gene expression. Structural diversity of hnRNPC between human/NWPs and mouse/rat/rabbit/dog was investigated by analysis of sequence variations within the hnRNP CLZ domain. The predicted loss of distal helical function in hnRNPC from lower species provides an explanation for the altered interaction between huC1/C2 and their mouse counterparts. These data provide new evidence of a role for $\mathrm{hnRNPC} 1 / \mathrm{C} 2$ in $1,25(\mathrm{OH})_{2} \mathrm{D}$-driven gene expression, and further suggest that species-specific tetramerization is a crucial determinant of its actions as a regulator of VDR-directed transactivation.

Bone Research (2014) 2, 14011; doi:10.1038/boneres.2014.11; published online: 01 July 2014

\section{INTRODUCTION}

1, 25-dihydroxyvitamin $\mathrm{D}\left[1,25(\mathrm{OH})_{2} \mathrm{D}\right]$ is the active form of vitamin $\mathrm{D}$ in target tissues such as bone. Upon interaction with its cognate intracellular receptor, vitamin $D$ receptor (VDR), and dimerizing with the unliganded retinoid $X$ receptor (RXR), the $1,25(\mathrm{OH})_{2} \mathrm{D}-\mathrm{VDR}-\mathrm{RXR}$ complex acts to transregulate the expression of genes by directly binding to specific vitamin D response elements (VDREs). This binding event influences transcription of vitamin Dresponsive genes via concerted interaction of the activated VDR with the chromatin remodeling apparatus as well as with receptor co-activators and co-repressors in the transcriptional machinery of the cell that ultimately control the diverse physiological actions of vitamin D. ${ }^{1-4}$ In previous studies we have described an additional regulatory component of intracellular $1,25(\mathrm{OH})_{2} \mathrm{D}-\mathrm{VDR}-\mathrm{RXR}$ signaling involving alternatively spiced members of the heterogeneous nuclear ribonucleoprotein $\mathrm{C}$ family of nucleic acid binding proteins (hnRNPl and hnRNP C)..$^{5-9}$

hnRNPs are abundantly expressed nucleocytoplasmic shuttle proteins that share a wide array of nucleic acid and protein targets that impact a multitude of cellular and molecular functions throughout the cell. ${ }^{10-12}$ For example, the functional hnRNPCl/C2 tetramer $\left(\mathrm{Cl}_{3} \mathrm{C}_{2}\right)^{13-14}$ is known to play a role in (i) chromatin remodeling: ${ }^{15}$ (ii) the early steps of spliceosome assembly and pre-mRNA splicing; $i^{16-17}$ and (iii) in modulating the stability, export and level of translation of bound mRNA molecules by interacting with the $5^{\prime}$-UTR of mRNAs and non-coding RNAs. ${ }^{18-19}$ Although classically characterized as a RNA binding and regulating protein, hnRNPCl/C2 can also interact with double-stranded DNA. In chromatin immunoprecipitation studies we have shown that hnRNPC1/C2 binds to VDREs in the absence of the VDR, with

'Department of Orthopaedic Surgery and the Orthopaedic Hospital Research Center, UCLA, Los Angeles, CA, USA ${ }^{2}$ Department of Regenerative Biology and Medicine, Mount Desert Island Biological Laboratory, Bar Harbor, ME, USA

Correspondence: M Hewison (mhewison@mednet.ucla.edu)

Received: 09 April 2014; Accepted: 24 April 2014 
hnRNPC1/C2-VDRE occupancy being displaced by the $1,25(\mathrm{OH})_{2}$ D-bound VDR-RXR complex. ${ }^{8}$ In this capacity hnRNPCl/C2 functions as a dominant-negative-acting VDRE-binding protein (VDRE-BP). This action of hnRNPCl/ C2 appears to contribute to normal VDR signaling capacity by occupying VDREs in the basal state and by participating in reciprocal cyclical VDRE binding following exposure to the VDR-activating ligand, $1,25(\mathrm{OH})_{2} \mathrm{D}$. This reciprocal relationship between VDR and hnRNPC1/C2 is disrupted when the VDRE-BP is overexpressed, leading to $1,25(\mathrm{OH})_{2} \mathrm{D}$ insensitivity in the target cell. ${ }^{8,20-23}$ In vivo, over-expression of hnRNPC1/C2 has been associated with vitamin D-resistant rachitic bone disease in vitamin D-deprived New World primates $^{24}$ and in man. ${ }^{7}$ Based on these molecular and phenotypic observations, the aim of the present study was to recapitulate and translate the VDRE-BP-induced vitamin D-resistant state in human bone cells by developing and characterizing murine osteoblastic cell lines which stably over express tetrameric HNRNPC proteins.

\section{MATERIALS AND METHODS}

Reagents and cell culture

Crystalline 1,25(OH) ${ }_{2} \mathrm{D}$ (Biomol, Plymouth Meeting, PA, USA) was reconstituted in absolute ethanol. Cells were treated with $0-10 \mathrm{nmol} \cdot \mathrm{L}^{-1}$ of $1,25(\mathrm{OH})_{2} \mathrm{D}$ incubated in the respective media with $1 \%$ FCS. MC3T3-El murine and MG63 human osteoblastic cells (both ATCC, Manassas, VA, USA) were maintained in $\alpha$-MEM plus $10 \% \mathrm{FBS}$ and incubated at $37{ }^{\circ} \mathrm{C}$ in a $95 \%$ air $/ 5 \% \mathrm{CO}_{2}$ atmosphere until $80 \%-90 \%$ confluent and then passaged. The J774A.1 mouse monocyte/macrophage cell line (ATCC) was cultured in DMEM with 10\% FCS. For overexpression studies, the hnRNPC1 and C2 constructs were previously cloned and ligated into the PCDNA 3.1/V5-HisTOPO vector (Invitrogen, Grand Island, NY, USA ${ }^{8}$ ); the constructs were transfected using the BioT reagent (Bioland Scientific, Paramount, CA, USA) in 12-well tissue culture plates $(0.1-1.0 \mu \mathrm{g}$ per well total recDNA). Cells were analyzed at designated time points post transfection or transduction.

\section{Cell fractionation and Western blot}

Treated cells were harvested and washed with ice-cold phosphate-buffered saline. Cell lysates were prepared in RIPA buffer with 1 X ProteoBlock protease inhibitor cocktail (Fermentas, Glen Burnie, MD, USA) and $1 \mathrm{mmol} \cdot \mathrm{L}^{-1} \mathrm{PMSF}$. For separation of nuclear and cytoplasmic fractions for extraction, the NE-PER nuclear and cytoplasmic extraction kit (Thermo Fisher Scientific Inc., Rockford, IL, USA) was utilized. Protein samples $(20 \mu \mathrm{g})$ were separated by SDS-PAGE. Primary anti-hnRNP C1/C2 (sc-32308; Santa Cruz Biotechnology, Santa Cruz, CA, USA) and secondary antibodies were used at 1:1000. I-Block (III) blocking reagent (Applied Biosystems, Foster City, CA, USA) was used during incubation steps.

Real-time quantitative PCR analysis

RNA was prepared using the RNeasy minikit (Qiagen Inc., Valencia, CA, USA). CDNA was synthesized by SuperScript Reverse Transcriptase III (Invitrogen) utilizing random hexamers. Quantitative PCR analysis was performed with a Stratagene MX-3005P instrument utilizing TaqMan ${ }_{\circledast}$ System reagents from Applied Biosystems (Supplementary Table S1). Target genes were normalized to 18 S rRNA expression. All CDNAs were amplified under the following conditions: $50{ }^{\circ} \mathrm{C}$ for $2 \mathrm{~min}$; $95{ }^{\circ} \mathrm{C}$ for $10 \mathrm{~min}$ followed by 40 cycles of $95{ }^{\circ} \mathrm{C}$ for $15 \mathrm{~s}$ and $60{ }^{\circ} \mathrm{C}$ for $1 \mathrm{~min}$. All reactions were performed in triplicate.

Bicistronic human hnRNPC 1 and $\mathrm{C} 2$ vector construction Construct pRRL-sin-cPPT-mouse (mu) collal $2.3 \mathrm{~kb}$ promoter-IRES-GFP-hPGK-puromycin was produced by PCR of mu pcollal with Xbal at the $5^{\prime}$ end (primer 5-collalXbal (Supplementary Table S2)) and blunted at the 3' end (primer 3-coll a l-Mlul) prior to digestion. The mouse coll a l $2.3 \mathrm{~kb}$ promoter was kindly provided by Benoit de Crombrugghe (Univ. Texas, M. D. Anderson Cancer Center). Construct pRRL-sin-cPPT-mu collal $2.3 \mathrm{~kb}$ promoter-human (hu) hnRNPCl-IRES-GFP-hPGK-puromycin was produced by PCR of hu hnRNPC ${ }^{8}$ with a Mlul site at the $5^{\prime}$ end (primer 5-hu hnRNPCl-Mlul) and EcoRl at the $3^{\prime}$ end (primer 3-hu hnRNPC 1-EcoRI). The products were then digested and ligated into PRRL-sin-cPPT-mu collal $2.3 \mathrm{~kb}$ promoter-IRES-GFP-hPGK-puromycin cut with Mlul and EcoRI. Construct pRRL-sin-cPPT-mu collal $2.3 \mathrm{~kb}$ promoter-hu hnRNPC 1-P2A-hu hnRNPC2-IRES-GFP-PGK-puromycin was produced by PCR of hu hnRNPC2 blunted at the $5^{\prime}$ end (primer 5-hu hnRNPC2) and with Clal at the $3^{\prime}$ end (primer 3-hu hnRNPC2-Clal). This product was then digested and ligated with PRRL-sin-CPPT-CMV-GFP-P2AMCS cut with Smal and ECoRI. This plasmid was then used to PCR P2A-hu hnRNPC2 blunted at the $5^{\prime}$ end (primer 5P2A) and with ECORI at the 3' end (primer 3-hu hnRNPC2ECORI). PCR of hu hnRNPCl was produced with EcoRl at the $5^{\prime}$ end (primer 5-hu hnRNPC 1-EcoRI) and blunted without the stop codon at the $3^{\prime}$ end (primer 3-hu hnRNPC 1$(-T A A))$. Both were digested and ligated into plasmid pRRL-sin-cPPT-mu collal $2.3 \mathrm{~kb}$ promoter-IRES-GFPhPGK-puromycin digested with EcoRI. Corresponding empty vectors were also constructed.

Lentiviral production and transduction

Lentiviral production was performed for construct pRRLsin-cPPT-mu collal $2.3 \mathrm{~kb}$ promoter-hnRNPC 1-IRES-GFPhPGK-puromycin. The construct PMD.G was used for the production of the VSV-G viral envelope in combination 
with the packaging constructs pHit $6 \mathrm{O}^{25}$ and the pBabe constructs correspond to the different transfer vectors. Briefly, $100 \mathrm{~mm}$ dishes of nonconfluent 293T cells were cotransfected with $6.5 \mu \mathrm{g}$ of pHit60, $3.5 \mu \mathrm{g}$ of pMDG (encoding the VSV-G envelope) and $10 \mu \mathrm{g}$ of $\mathrm{p}$ Babe (hu hnRNPCl construct), by the CaPi-DNA coprecipitation method. ${ }^{26}$ Next day, the medium was adjusted to make a final concentration of $10 \mathrm{mmol} \cdot \mathrm{L}^{-1}$ sodium butyrate and the cells were incubated for $8 \mathrm{~h}$ to obtain high-titer virus production as previously described. After the 8-h incubation, cells were washed and incubated in fresh medium without sodium butyrate. Conditioned medium was harvested $16 \mathrm{~h}$ later and passed through $0.45 \mathrm{~mm}$ filters. For transduction of MC3T3-El cells, cells were seeded at $8 \times 10^{4}$ cells per well in 12-well plates. Transductions were carried out in $0.5 \mathrm{~mL}$ DMEM, including serial dilutions of lentiviral vector supernatant. The cells were then washed with phosphate-buffered saline after $24 \mathrm{~h}$ post transduction and incubated in regular medium for $48 \mathrm{~h}$.

Molecular dynamic simulations and free energy calculations for hnRNPC

The coordinates of the human hnRNP C leucine zipper-like oligomerization domain (CLZ) tetramer (PDB code 1TXP) ${ }^{27}$ were used for the simulations. PDB structure contains lle at position $180 ;{ }^{27}$ to get the correct human or mouse sequence, the lle was mutated to Leu using the program PyMol (http://www.pymol.org). The mouse hnRNPC tetramer was built by mutating the residue Asn200 to Ser. Molecular dynamics simulations were performed in explicit water using NAMD ${ }^{28}$ with CHARMM27/CMAP force field. ${ }^{29}$ The tetramers were solvated in a cubic TIP3P solvent box of $9.0 \AA$ A. Periodic boundary conditions, Particle Mesh Ewald summation and SHAKE-enabled 2-femto seconds time steps were used in all molecular dynamics simulations. Initial configurations were subjected to a 500-step minimization with the harmonic constraints of $10 \mathrm{kcal} \cdot \mathrm{mol}^{-1} \cdot \AA^{-2}$ on the protein heavy atoms. The systems were gradually heated from 0 to $300 \mathrm{~K}$ over a period of $30 \mathrm{ps}$ with harmonic constraints. The simulation at $300 \mathrm{~K}$ was then continved for 200 ps during which the harmonic constraints were gradually lifted. The systems were then equilibrated for a period of 300 ps before the 10 ns production run. All simulations were carried out in the NPT ensemble. The binding free energy of the hnRNPC2 to hnRNPC1 for the different (interspecies) combinations was estimated using the MMPBSA module in AMBER 9.0 (Ref. 30) by taking snapshots (2 000) at every 5 ps from the 10-ns production run.

Data normalization and statistical analysis

The real-time quantitative PCR methodology is based on determining the delta cycle threshold $\left(\Delta C_{t}\right)$ values. The average $\Delta C_{\dagger}$ value was taken for triplicate sets.
The fold induction was calculated by the formula: $2^{\left(\operatorname{avg} \Delta C_{+} 0 \mathrm{~nm}-\operatorname{avg} \Delta C_{+} i\right)}$, where $i$ is $0-10 \mathrm{nmol} \cdot \mathrm{L}^{-1}$. An analysis of variance statistical test was conducted with a Bonferroni multiple comparison post hoc analysis where $P \leq 0.05$ was considered statistically significant.

\section{RESULTS}

Effects of human hnRNPC $1 / \mathrm{C} 2$ on $1,25(\mathrm{OH})_{2}$ D-mediated gene transactivation in mouse osteoblasts

As shown in Figure 1a, when transfected into mouse MC3T3 osteoblasts, suppression of $1,25(\mathrm{OH})_{2} \mathrm{D}$-mediated transcription was only observed when huCl and huC2 were used in combination. In fact, expression of Ddit4, a gene we have previously shown to be induced by $1,25(\mathrm{OH})_{2} \mathrm{D}$ in osteoblasts, ${ }^{20}$ was enhanced not suppressed, in mouse MC3T3 cells transfected with either huCl or huC2 alone in the presence of $1,25(\mathrm{OH})_{2} \mathrm{D}$. By contrast, overexpression of either huCl or huC2 was sufficient to suppress $1,25(\mathrm{OH})_{2} \mathrm{D}$ induced gene expression in human MG-63 osteoblast-like cells, with combined over-expression of huCl and huC2 having an additive suppressive effect (Figure 1b).

To determine whether the lack of efficacy of huCl and huC2 individually to squelch $1,25(\mathrm{OH})_{2} \mathrm{D}$-directed gene expression was due to relatively low transfection efficiency and subsequent expression of the human subunits in mouse osteoblasts, similar studies were also carried out using a bone-specific vector to drive expression of huCl in mouse cells harboring the endogenously expressed oligomerization partner, muC2 (Figure $2 a$ and $2 b$ ). Despite 50- to 3000 -fold higher levels of expression relative to the endogenous $\mathrm{muC} 1$ and muC 2 expression levels, transfection with huCl continued to have no significant effect on $1,25(\mathrm{OH})_{2} \mathrm{D}$-induced gene expression (Figure $2 \mathrm{C}$ ).

The apparent requirement for both huCl and huC2 to suppress $1,25(\mathrm{OH})_{2} \mathrm{D}$-directed transcription in mouse osteoblasts was further illustrated in MC3T3-transfected cell lines where huC 1 and huC2 were co-expressed under the control of the osteoblast-specific mouse collal promoter. In contrast to the previous experiments, vectors used in this part of the study encoded the huCl and huC2 linked to one another by the self-cleaving 23 amino-acid picornaviral 2A-like sequence from the porcine teschovirus-1 (P2A) to allow for efficient functional stoichiometric expression of multiple flanking proteins under the influence of the bone-specific coll al $2.3 \mathrm{kB}$ promoter (Figure $3 \mathrm{a}^{31}$ ). The advantage of combined transgene expression with the P2A over transgenes linked by an internal ribosomal entry site or introduced by individual constructs is its independence of copy number required for integration, its relatively small size and its high cleavage efficiency. ${ }^{32}$ Using this system, both the huCl and huC2 isoforms were equally overexpressed specifically in the nuclear compartment of MC3T3 cells (Figure 3b). The 

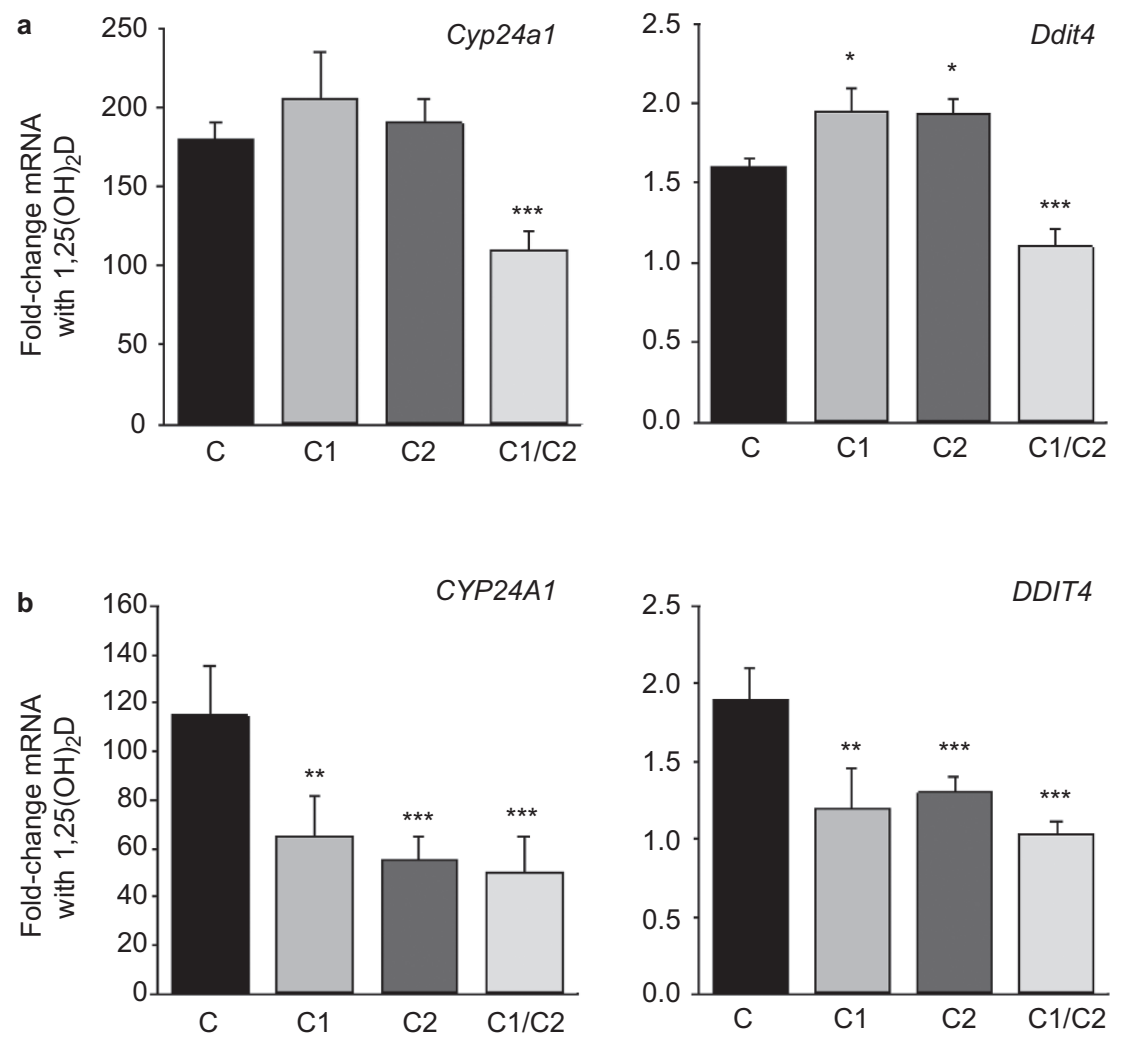

Figure 1. Effects of overexpressed human hnRNPC1 and C2 on VDR-mediated gene transcription in human and mouse osteoblast-like cells. Effect of overexpression of human hnRNPC1 (C1) and/or human hnRNPC2 (C2) on 1,25(OH) ${ }_{2} \mathrm{D}$-induction of the vitamin D-target genes CYP24A1/Cyp24a1 and DDIT4/Ddit4 in (a) mouse MC3T3 osteoblastic cells and (b) human MG-63 osteoblastic cells. Cells were treated with or without $10 \mathrm{nmol} \cdot \mathrm{L}^{-1}$ $1,25(\mathrm{OH})_{2} \mathrm{D}$ for $6 \mathrm{~h}, 24 \mathrm{~h}$ post transfection with either empty vector $(\mathbf{c}), \mathrm{C} 1, \mathrm{C} 2$ or $\mathrm{C} 1$ and $\mathrm{C} 2(\mathrm{C} 1 / \mathrm{C} 2)$. Data show mean \pm s.d. ( $n=3$ separate cultures) fold-change in mRNA expression for CYP24A1/Cyp24a1 and DDIT4/Ddit4 for each transfected cell type following treatment with 1,25(OH) ${ }_{2} \mathrm{D}$ relative to vehicle-treated control cells. $* * * P<0.001$, statistically different from empty vector controls $(\mathrm{C})$, **P<0.01, statistically different from $\mathrm{C}$.

efficiency of transfection of the single open reading frame was assessed by green fluorescent protein (GFP) expression within various cell lines (Figure $4 \mathrm{a}$ ), and the absence of uncleaved product in Western blot analyses indicated efficient cleavage of the P2A protein (Figure 4b). Cell (tissue)specific expression of huCl/huC2 was confirmed by parallel analysis of the temporal pattern of endogenous mouse Collal mRNA levels over time in MC3T3 bone cells, but not J774A mouse monocytes (24-96 h) (Figure 5).

As shown in Figure 3c, co-expression of huCl and huC2 using the $\mathrm{P} 2 \mathrm{~A}$ vector acted as a dominant-negative inhibitor of $1,25(\mathrm{OH})_{2} \mathrm{D}$-induced expression of Cyp24al and Ddit4 target genes in mouse osteoblasts. Endogenous muCl/muC2 transcript levels were unchanged under both basal and 1,25(OH) ${ }_{2}$ D-treated conditions (Figure 6), suggesting no interference by endogenous mouse hnRNPC protein subunits.

Species-specific variations in the structure of the hnRNPC oligomerization domains dictate function

The hnRNPC proteins associate with RNA as tetramers with a composition of three hnRNP Cl subunits and one hnRNP C2 subunit $\left(\mathrm{Cl}_{3}-\mathrm{C}_{2}\right)$. Studies in vivo have shown that the formation of the tetrameric complex, which is highly stable in solution, is driven by a 28-residue helical region (residues 180-207) referred to as hnRNPC leucine zipper-like (CLZ) oligomerization domain ${ }^{27}$ (Figure 7a). The CLZ domain promotes the oligomerization by forming a coiled coil tetramer structure; hnRNPC proteins possessing mutations in the CLZ domain bind weakly to nucleic acid substrates. ${ }^{27}$ Amino-acid sequences of the hnRNPC CLZ oligomerization domain are well conserved within vertebrates, exhibiting 74\%-98\% identity to the human CLZ domain (Figure 7b). Like many oligomerizing proteins, hnRNPC isoforms employ $\alpha$-helical coiled coils as an oligomerization mechanism with the parts stabilized by continuous interhelical contacts formed between hydrophobic faces of amphipathic helices. ${ }^{33}$ For each set of seven residues (heptad) along the helix (denoted a-g; Figure 7b), the contact geometry is repeated for the ideal coiled coil. Hydrophobic residues occupying the a and $d$ heptad positions form interhelical contacts in the coiled coil core as well as other contact surfaces at interfacial e and $g$ heptad positions. Comparison of the human and mouse hnRNPC sequences in Figure $7 b$ revealed that the Ser at position 200 in the mouse is replaced by Asp in man; as 
a

\begin{tabular}{|l|l|l|l|l|l|l|l|}
\hline RRL & cPPT & mouse pcol1a1 $(2.3 \mathrm{kB})$ & (hu)hnRNPC1 & IRES & GFP & mPGK & puromycin \\
\hline
\end{tabular}

b
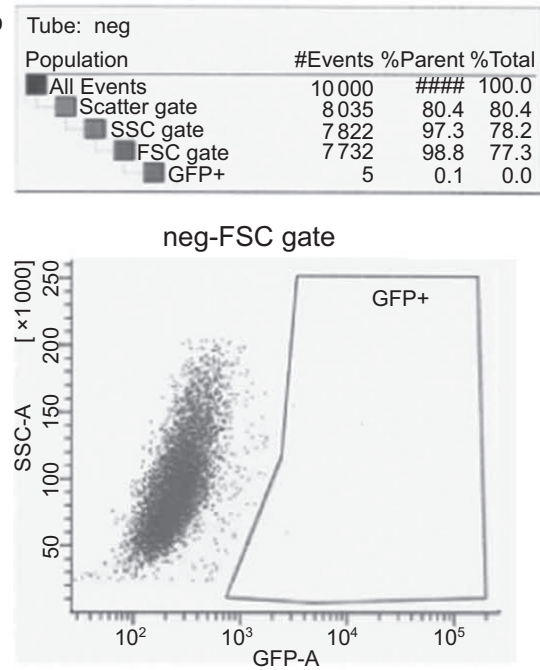

c
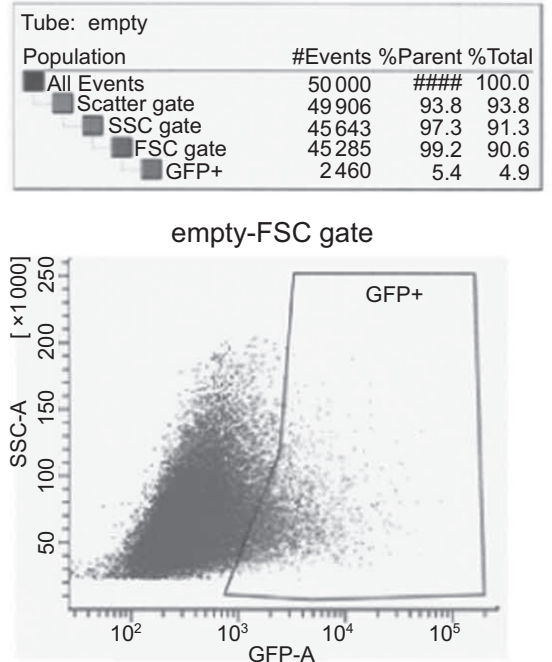

hu HNRNPC1 clone set (MC3T3-E1)
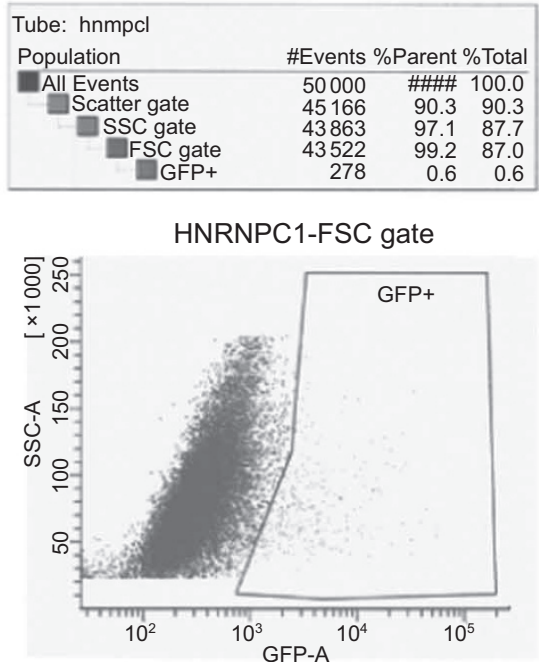

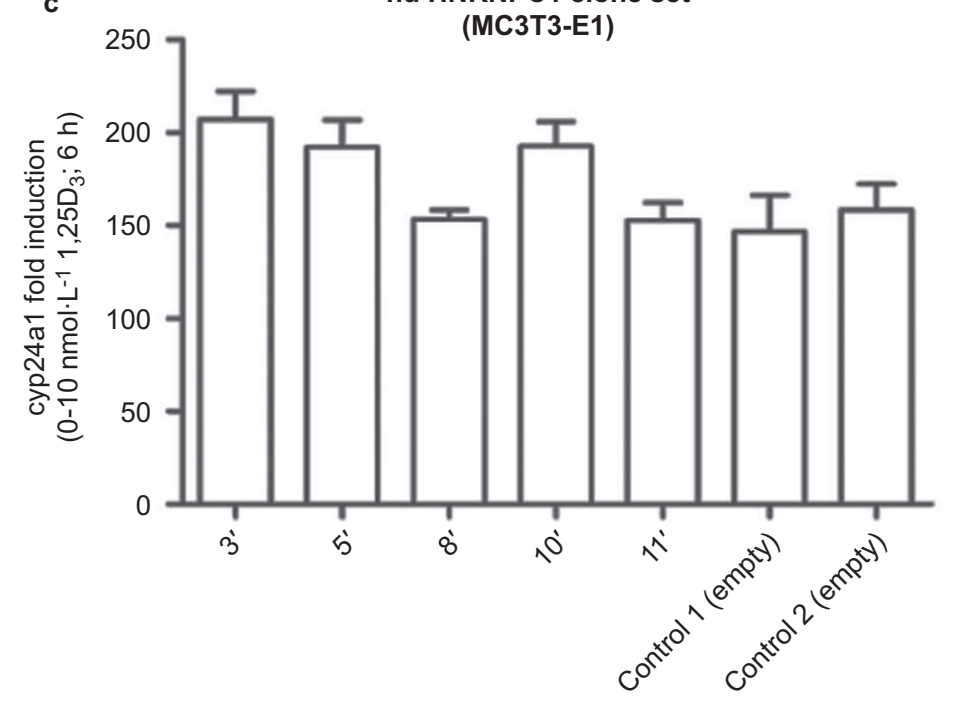

Figure 2. Stable expression of only human hnRNPC1 protein in mouse MC3T3-E1 osteoblasts does not confer resistance to 1,25(OH) ${ }_{2} \mathrm{D}$. (a) Osteoblastic transduction was performed using the lentivirus RRL transfer vector harboring a stop codon-less human (hu) hnRNPC1 cDNA and a GFP cassette driven by the bone-specific mouse col1a1 $2.3 \mathrm{kB}$ promoter. The lentivector backbone also contains the cPPT from the HIV-1 integrase gene to increase the copy number of lentivirus integrating into the host genome to enhance viral titer. (b) FACS sorting of GFP-positive MC3T3-E1 osteoblasts transduced with the hu hnRNPC1 and empty lentivirus vectors for the isolation and generation of stable clones. (c) Fifteen hu hnRNPC1-overexpressing stable lines were created (data not shown), whereby five (clones $3^{\prime}, 5^{\prime}, 8^{\prime}, 10^{\prime}, 11^{\prime}$ ) lines with variable hnRNPC1 overexpression were further evaluated for $1,25(\mathrm{OH})_{2} \mathrm{D}\left(10 \mathrm{nmol} \cdot \mathrm{L}^{-1}, 6 \mathrm{~h}\right)$ mediated induction of endogenous mouse $(\mathrm{mu})$ Cyp24a1 mRNA. cPPT, central polypurine tract.

such, the most distal interfacial g position heptad of the hnRNPC CLZ domain used for hnRNPC1-hnRNPC2 oligomerization in the mouse is disrupted.

Free energy and stability analysis of tetramers formed by combinations of mouse and human hnRNPC $1 / C 2$

To determine whether interaction between the Asp-200 in the human and Ser-200 in the mouse hnRNPC protein was sufficient to cause destabilization of the CLZ-coiled hnRNPC1/C2 tetramer, the binding free energy of interspecies tetramers harboring three hnRNPCl and one hnRNPC2 subunit combinations, huCl $1_{3}-\mathrm{muC}_{2}$ and muCl $1_{3}-$ huC2 $2_{1}$, was calculated using the MMPBSA method implemented in Amber 9.0. When calculating the binding free energy, the holotetramer was considered as a complex, the three hnRNPCl subunits as a receptor and the 
b

mouse pcol1a1 (2.3kB) (hu)hnRNPC1 $\mathrm{P} 2 \mathrm{~A}(\mathrm{hu}) \mathrm{hnRNPC2}$ |RES GFP mPGK puromycin
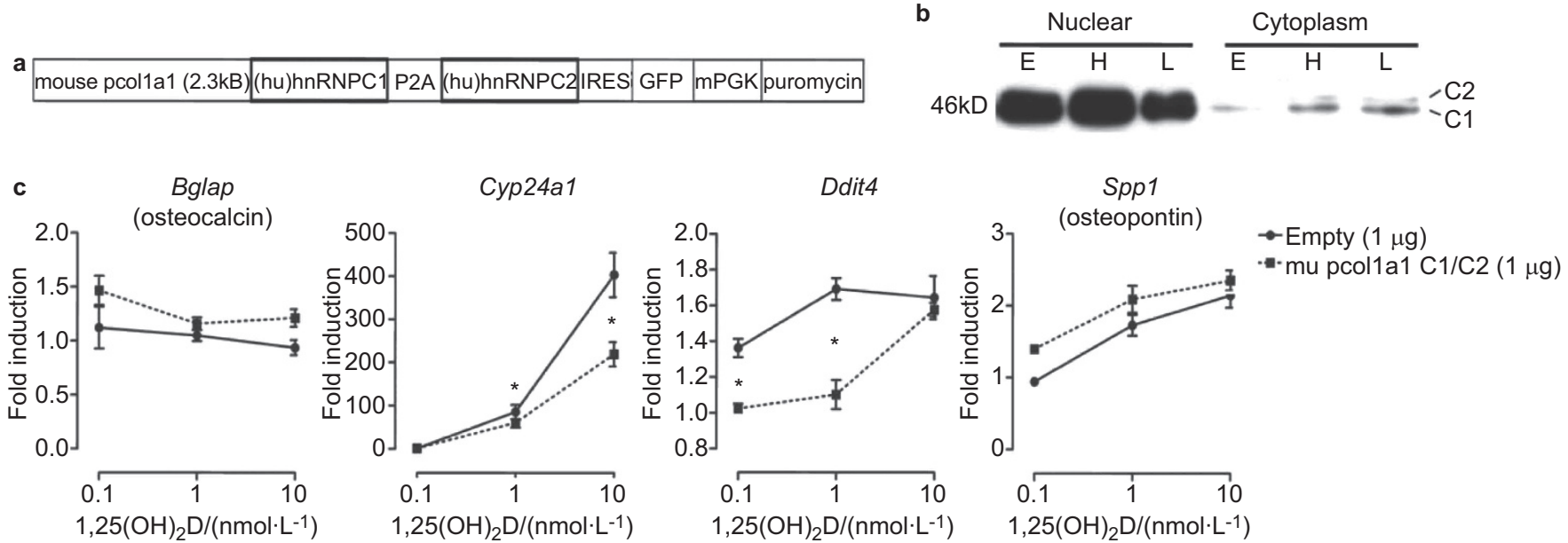

Figure 3. Equimolar expression of human hnRNPC1 and C2 confers resistance to 1,25(OH) $2 \mathrm{D}$ in mouse MC3T3-E1 osteoblasts. (a) Schematic representation of the mouse colla1 $2.3 \mathrm{kB}$ promoter-driven piconoviral P2A sequence construct for the co-expression of human (hu) hnRNPC1 and C2 proteins with a GFP reporter. (b) Western blot analyses showing overexpression of hnRNPC1 and C2 proteins using the P2A construct enriched in the nuclear fraction of MC3T3-E1 cells at $96 \mathrm{~h}$ post transfection. Empty vector (E), and high (H, $2 \mu \mathrm{g}$ per well) and low (L, $0.1 \mu \mathrm{g}$ per well) levels of P2A expression vector cDNA were transfected into MC3T3 cells. (c) $1,25(\mathrm{OH})_{2} \mathrm{D}\left(0.1-10 \mathrm{nmol} \cdot \mathrm{L}^{-1} ; 6 \mathrm{~h}\right)$ induction of mouse osteoblastic genes after transfection of empty vector or hnRNPC1/C2 P2A expression construct in MC3T3-E1 96 h post transfection.

hnRNPC2 subunit and its CLZ domain as a binding ligand partner. In all cases, the negative binding free energy indicated favorable interactions between the $\mathrm{Cl}_{3}$ and $\mathrm{C}_{1}$
CLZ domains regardless of the species derivation of the subunits. As shown in Table 1, the highest negative binding energy was observed with huCl$l_{3}-h u C 2_{1}$, possibly due to
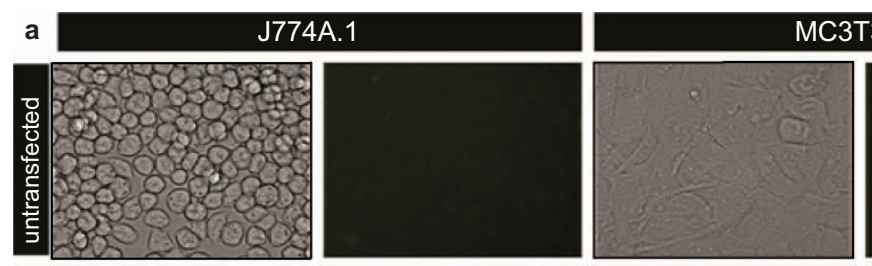

MC3T3-E1
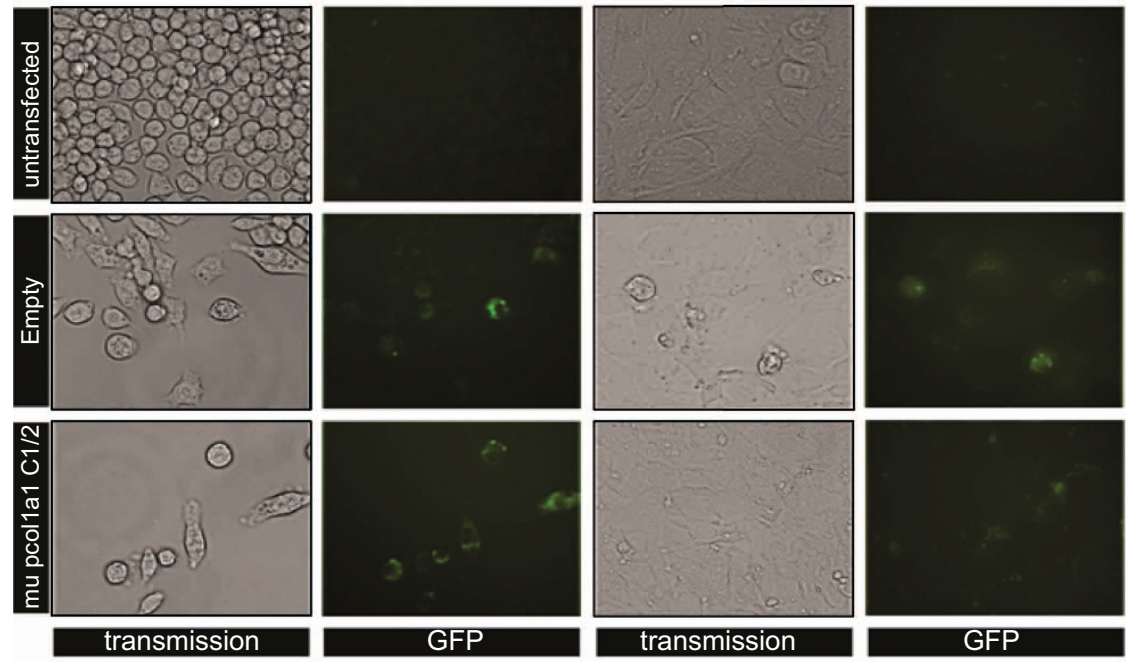

b

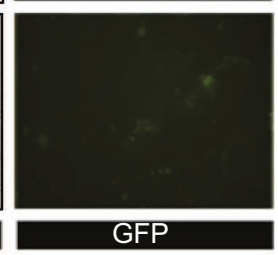

J774A.1

(96 hours)

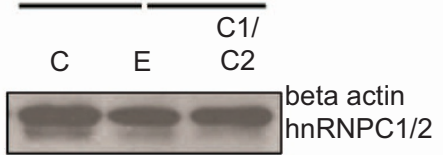

MC3T3-E1

(96 hours)

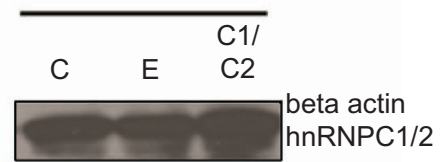

Figure 4. Efficient transfection of the P2A hnRNPC1/C2 construct. (a) Transient transfection of P2A hnRNPC1/C2 construct with IRES-GFP reporter shows efficient labeling of both J774A.1 mouse monocytic and MC3T3-E1 osteoblastic cells after $96 \mathrm{~h}$ post transfection. The empty vector control also depicts efficient and comparable GFP labeling. (b) Western analysis of whole cell lysate for beta-actin and hnRNPC1/C2 expression $96 \mathrm{~h}$ post transfection. 

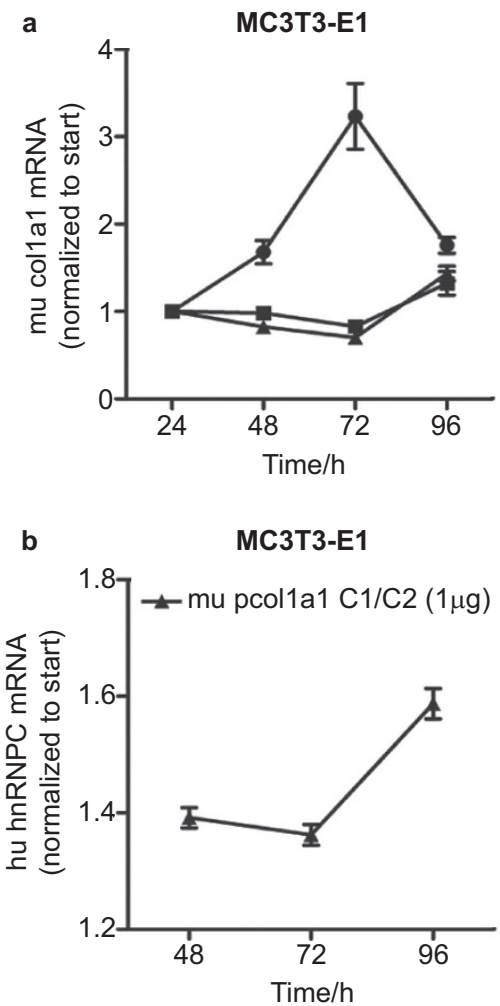

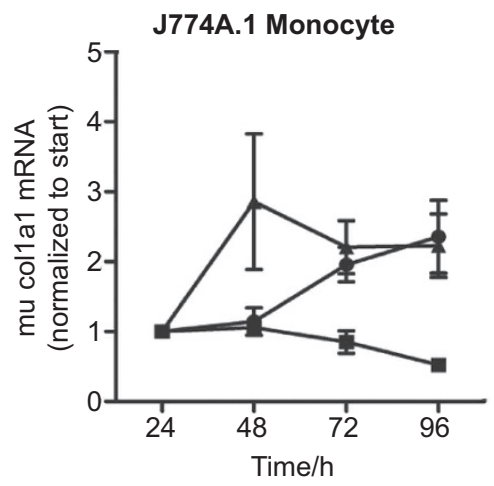

$\rightarrow$ Untransfected

$\rightarrow$ Empty $(1 \mu \mathrm{g})$

- mu pcol1a1 C1/C2 (1 $\mu \mathrm{g})$

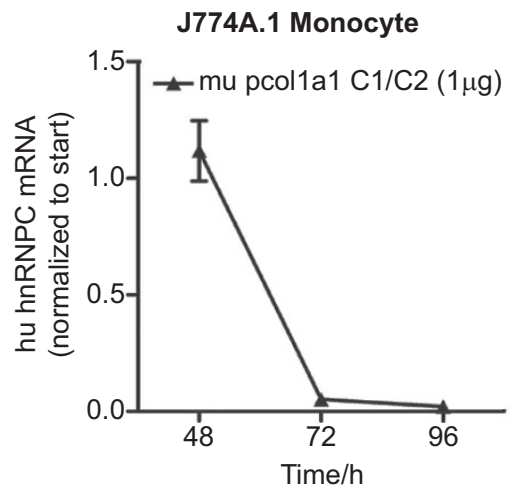

c

hu hnRNPC1/C2

mRNA

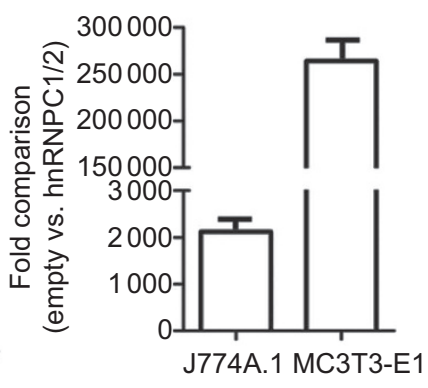

Figure 5. Cell type-specific action and temporal regulation of the P2A hnRNPC1/C2 construct. (a) Endogenous mouse (mu) type I collagen expression was monitored over a 96-h period under the different transient transfection conditions. For both transfected constructs into MC3T3-E1 cells, col1a1 mRNA expression was delayed when compared to the untransfected controls. In J774A.1 mouse monocytes the expression of endogenous mu type I collagen was variable between the transfected P2A construct and untransfected control, and unregulated in the empty vector control. (b) Human (hu) hnRNPC mRNA levels were monitored in the mouse col1a1 promoter-driven P2A construct-transfected cells at $96 \mathrm{~h}$ post transfection. Hu hnRNPC mRNA was temporally regulated in a similar fashion as mu type I collagen in MC3T3-E1 osteoblasts. No temporal regulation of huhnRNPC mRNA was observed in J774A.1 mu monocytes transfected with the P2A construct, suggesting the mu col1a1 $2.3 \mathrm{kB}$ promoter was bone-specific. (c) After $96 \mathrm{~h}$ of transfection, MC3T3-E1 cells harboring the P2A hnRNPC1/C2 construct express huhnRNPC with mRNA levels approximately 125-fold higher relative to the matching empty vector control when compared to the J774A.1 mouse monocytic cell line.

the additional favorable interactions that could occur between Asn-200 and Glu-186 in the tetramer (Figure 7c, left panel). By contrast, only one hydrogen bond is possible

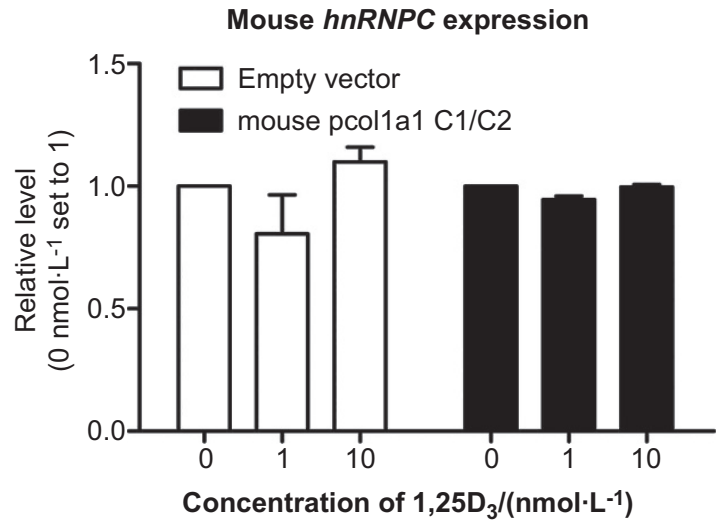

Figure 6. Endogenous mouse hnRNPC not responsible for functional effects. Endogenous mouse (mu) hnRNPC expression was monitored in MC3T3-E1 cells after transfection and 6-h treatment with various concentrations of $1,25(\mathrm{OH})_{2} \mathrm{D}$. for the $\mathrm{muCl}_{3}-\mathrm{huC2}{ }_{1}$ complex (Figure 7c, right panel), providing a potential explanation for the relatively low negative binding energy for the mouse hnRNPC1/C2 tetramer. The negative free energy of the interspecies oligomeric combinations, $\mathrm{muCl}_{3}-\mathrm{huC2}{ }_{1}\left(-107.22 \mathrm{kcal} \cdot \mathrm{mol}^{-1}\right)$ and huCl $1_{3}-\mathrm{muC2}_{1}\left(-103.25 \mathrm{kcal} \cdot \mathrm{mol}^{-1}\right)$, were intermediate to the species-specific tetramers (Table 1). These results indicate that the Asn-200 in the human hnRNPC CLZ domain plays a pivotal role in stabilization of the hnRNPCl/C2 oligomer in man. To assess the potential impact of hnRNPC subunit binding energy variations on tetramer stabilization, molecular dynamic simulations were carried out (Figure 7d). Data are shown as the root mean square deviations for the backbone atoms ( $\mathrm{N}-\mathrm{CA}-$ $\mathrm{C}-\mathrm{O})$ from the experimental structure, over $10 \mathrm{~ns}$ for the four tetrameric combinations depicted in Table 1. The huCl $1_{3}-$ muC2 $_{1}$ interspecies tetramer complex showed large fluctuations (instability) during the initial phases of the 10-ns period of simulation, but eventually reached a state comparable the other tetramers after $7 \mathrm{~ns}$. 
a

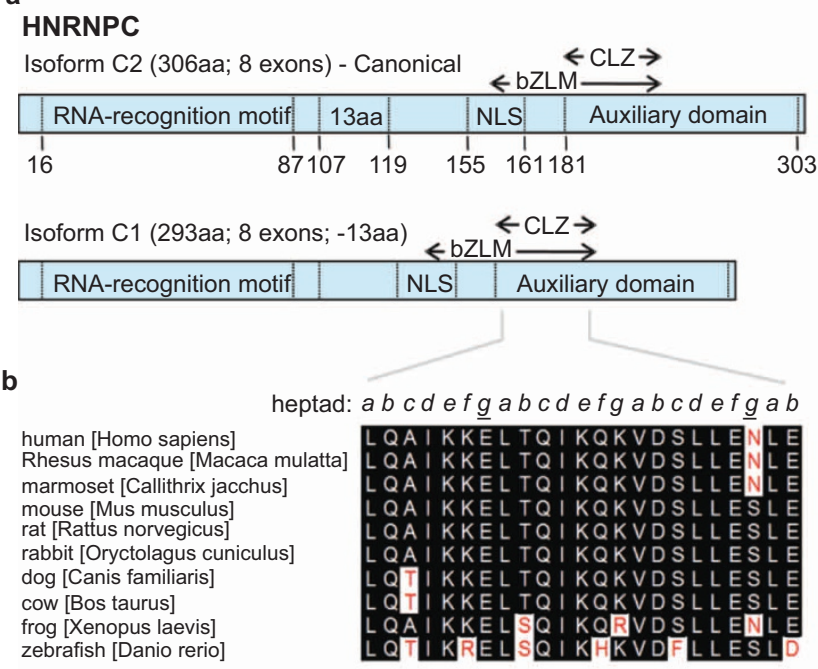

c

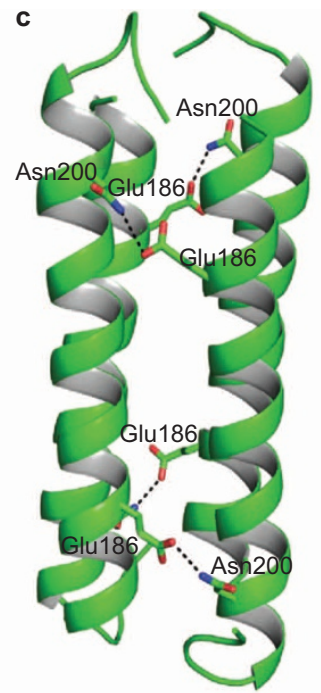

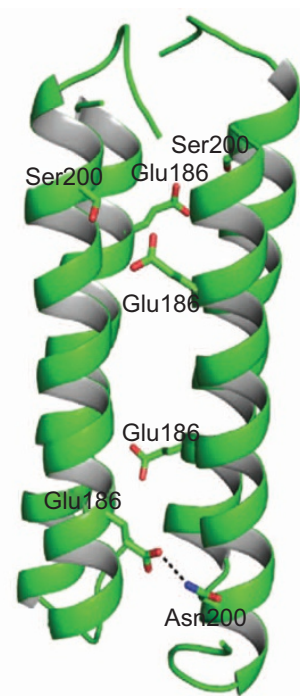

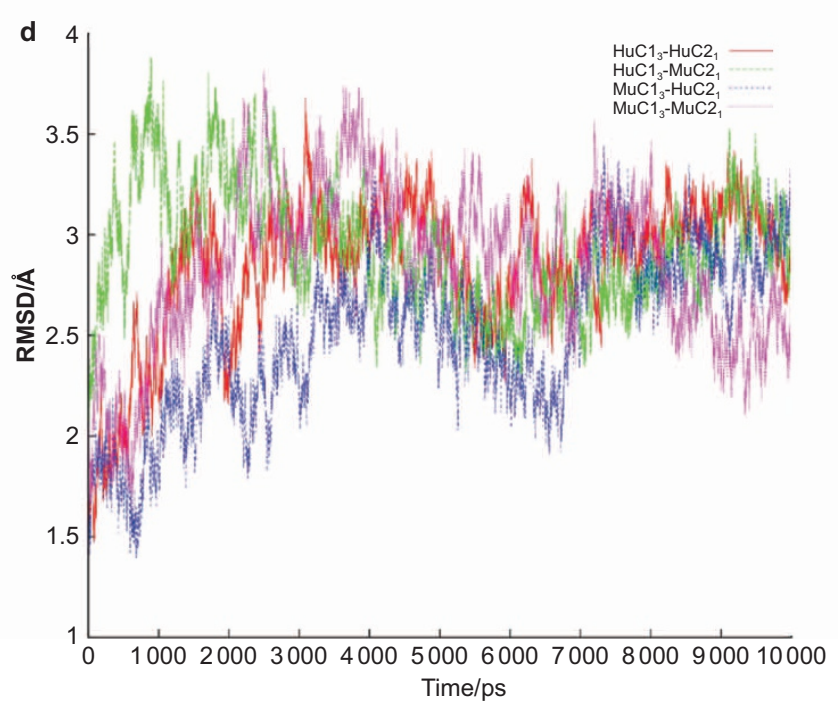

Figure 7. hnRNPC1/C2 tetramer stability based on species-specific amino-acid substitutions. (a) Schematic representation of human hnRNPC isoforms. Each protein contains a single RRM, a delineated NLS, a bZLM and an encompassing acidic auxiliary domain. hnRNPC2 contains an additional 13 aa and is expressed at one-third the level of hnRNPC1. An oligomerization domain (CLZ) is also present in C proteins. Numbers represent aa position. (b) Alignment of CLZ sequences derived from the Ensembl database (http://www.ensembl.org). Key asparagine (N) to serine (S) change within the mouse sequences occupying an interhelical contact surface heptad residue within the coiled coil core of hnRNP C. Similar residues are highlighted in black, while discrepant residues are highlighted in white. The heptad arrangement is in italics, with the key intermolecular residues forming antiparallel chain contacts underlined and bolded. Numbering based on the human C2 full-length isoform. (c) The hnRNPC protein CLZ tetramers of $\mathrm{Hu}$ and $\mathrm{Mu} \mathrm{C} 1$ and $\mathrm{C} 2$ proteins. $\mathrm{HuC1}_{3}-\mathrm{HuC}_{1}$ (left panel) and $\mathrm{MuC1}_{3}-\mathrm{HuC}_{1}$ (right panel). The Asn200, Ser200 and Glu186 residues are shown in stick representation. In $\mathrm{HuC}_{3}-\mathrm{HuC}_{1} \mathrm{CLZ}$ domains, pairs from the adjacent helices make four hydrogen bonds in the coiled coil tetramer. In contrast, in the $\mathrm{MuCl}_{3}-\mathrm{HuC}_{1}$ tetramer, only one hydrogen bond is possible. The hydrogen bonds formed between Asn200 and Glu186 are shown in dashed lines. (d) Molecular dynamic simulations for CLZ tetramers. The root mean square fluctuations for the backbone atoms of the tetramer complexes for different combinations of human $(\mathrm{Hu})$ and mouse $(\mathrm{Mu}) \mathrm{hnRNPC1}$ and $\mathrm{C} 2 . \mathrm{HuC1}_{3}-\mathrm{HuC2}_{1}, \mathrm{red} ; \mathrm{HuC1}_{3}-\mathrm{MuC2}_{1}, \mathrm{green} ; \mathrm{MuC1}_{3}-$ $\mathrm{HuC2}_{1}$, blue; and $\mathrm{MuC1}_{3}-\mathrm{MuC2}_{1}$, magenta. Data are based on experimental structure over 10-ns simulations. bZLM, basic leucine zipper-like motif; NLS, nuclear localization signal; RRM, RNA recognition motif.

\section{DISCUSSION}

The goal of the present study was to develop a mouse model that recapitulated the hnRNPC1/C2 (VDRE-BP) overexpression-induced vitamin D-resistant state observed in vivo in the skeletons of growing nonhuman and human primates. ${ }^{7}$ Before developing the animal model, we assessed the effects of hnRNPCl/C2 overexpression using a mouse osteoblast cell line. The studies here show that overexpression of huCl and huC2 in combination or transfection with a bone-specific, polycistronic vector suppressed transspecies $1,25(\mathrm{OH})_{2} \mathrm{D}$ mediated induction of mouse osteoblast target gene expression. This finding suggests that human hnRNPCl/ C2 can function as a dominant-negative-acting inhibitor 
Table 1. Binding free energy of the hnRNPC CLZ domain according to tetramer combinations of mouse and human hnRNPC1 and C2. The total binding free energy for different combinations of human (hu) and mouse (mu) hnRNPC1 and C2 CLZ domains, estimated from MMPBSA calculations

\begin{tabular}{ll}
\hline $\mathrm{CLZ}$ tetramer & $(\Delta \mathrm{GMM} / \mathrm{PBSA}) /\left(\mathrm{kcal} \cdot \mathrm{mol}^{-1}\right)$ \\
\hline $\mathrm{HuCl}_{3}-\mathrm{HuC2}{ }_{1}$ & -112.07 \\
$\mathrm{HuCl}_{3}-\mathrm{MuC2}_{1}$ & -103.25 \\
$\mathrm{MuC1}_{3}-\mathrm{HuC2}_{1}$ & -107.22 \\
$\mathrm{MuCl}_{3}-\mathrm{MuC2}_{1}$ & -94.59 \\
\hline
\end{tabular}

of $1,25(\mathrm{OH})_{2} \mathrm{D}$-driven gene expression in mouse bone cells and that species-specific tetramerization is a critical determinant of its actions as a regulator of VDR-directed transactivation.

A monomer of the hnRNPC protein has a primary structure that is common to hnRNPs and which can be divided into four distinct domains: (i) the RNA recognition motif consisting of beta-sheets; (ii) the basic high affinity RNA binding domain or basic leucine zipper-like motif, residues 180-207; (iii) the leucine zipper containing four heptad repeats toward the carboxyl-terminus (CLZ); and (iv) the negatively charged carboxyl-terminal domain (CTD). ${ }^{34-35}$ It is the basic leucine zipper-like motif domain, rather than the RNA recognition motif, which plays the major role in binding to pre-mRNA and snRNA with high affinity. ${ }^{36}$ Based on detailed sequence-specific labeling and solution structural analyses, the current model suggests that hnRNPC proteins exist in an anti-parallel four-helix tetrameric bundle which is centrally positioned and that these bundles assemble through highly stable associations of their leucine zipper CLZ domains. ${ }^{27,37}$ The human and mouse CLZ domains are well conserved with the only difference being an Asp to Ser change at the terminal helix of the coiled coil structure (Figure 7b). The CTD is thought to be involved in protein-protein interactions that serve to increase specificity of nucleotide binding. Although there is increasing awareness of the composition, function and mechanisms associated with hnRNPC and homologous proteins, the protein-protein interactions that participate in their recruitment to cis elements along genomic DNA have yet to be fully defined. Emerging evidence shows that the CTD of hnRNPs interacts with transcription elongation factors, chromatin-modifying complexes and pre-mRNA maturation enzymes, thus providing a molecular basis for the coupling between gene transcription and RNA processing. ${ }^{15}$ While the hnRNPC protein domain responsible for interaction in cis with VDREs regulated expression of $1,25(\mathrm{OH})_{2} \mathrm{D}$-driven target genes remains uncertain, our modeling indicates that the DNA transbinding domain may be located within a hinge portion of the acidic CTD. This would place it apart from the core bundle stabilization domain known to bind cis elements which vary among higher and lower mammalian species. ${ }^{15,37}$
hnRNPs were first recognized for their ability to bind to single-stranded RNA. ${ }^{10,38-39}$ However, it was subsequently determined that hnRNPs have the ability to bind both single- and double-stranded DNA ${ }^{5,7,15}$ with specific cis-acting DNA sequences being important determinants in transcriptional regulation of gene expression under the control of steroid hormone receptors such as VDR and the estrogen receptor. ${ }^{9}$ In the current study, overexpression of either huC 1 or huC2 in human osteoblasts was sufficient to suppress $1,25(\mathrm{OH})_{2} \mathrm{D}$-VDR-mediated signaling. Unexpectedly, repression of $1,25(\mathrm{OH})_{2} \mathrm{D}-\mathrm{VDR}$-VDR-directed transcription was only achieved by co-overexpression of both huC 1 and huC2 in murine osteoblasts. These data, together with molecular dynamic simulation analyses presented in Figure 7, suggest that optimal DNA binding and the subsequent dominant-negative action of hnRNPCl/ C2 on transcription occurs with species-specific tetramerization patterns involving the $\mathrm{C} 1$ and $\mathrm{C} 2$ isoforms (Figure 7). Binding free energy modeling further suggests that this differential response is due to subtle variations in the protein-protein interactions required for hnRNPC 1/C2 tetramerization.

While the computational molecular dynamic studies presented here showed that interspecies tetramers of $\mathrm{Cl}$ and C2 can form (Figure 7), it is also known that tetramer stability is influenced by elements in the negatively charged CTD of hnRNPC that have diverged most between mouse and man. ${ }^{36}$ For example, a deletion construct of the human hnRNPC lacking the CTD beyond the leucine zipper can exist as a dimer, ${ }^{37}$ highlighting the importance of this region of hnRNPC protein for tetramer assembly. Further, velocity sedimentation analysis of deletion constructs of the hnRNPC CTD demonstrates that the residues between S-240 and G-263 play a critical role in the native tetramer formation and stabilization. ${ }^{37}$ Interestingly, the only proximal variation between mouse and human sequences within the CTD is at amino-acid position 242 , where mouse harbors an alanine and humans a glycine (data not shown), suggesting that differences in the hydropathy index of that portion of the CTD may alter CTDdependent functions. Importantly, site-specific mutagenesis experiments that either removed or reversed the charges at specific residues within this proximal CTD resulted in a decrease in tetramer assembly. ${ }^{37}$ Either lysine or alanine (non-charged) substitutions significantly attenuated tetramer assembly; the mouse harbors an alanine at position 242 in the CTD of hnRNPC. These observations suggest that the incompletely conserved CLZ domain and the carboxyl-terminal half of the CTD between mouse and man may disturb the function of hnRNPC by affecting subunit oligomerization and by altering interactions between the non-homologous residues of the CTD and the four-helix bundle of native hnRNPC proteins. With these observations 
in mind, we postulate that changes in the helix-interaction structures of hnRNPC tetramers can fundamentally alter its binding affinity for cis elements controling $1,25(\mathrm{OH})_{2} \mathrm{D}-$ VDR-driven target genes.

It is clear that the full range of hnRNPC activities has not been elucidated. For example, members of the hnRNPC family can bind single-stranded DNA, ${ }^{40}$ suggesting that they may have distinct roles in DNA replication and recombination events as well as in transcription and transcript handling. The identification of species-specific cellular functions of hnRNPC proteins and their specific DNA targets will greatly help to elucidate the broader role of this versatile nuclear protein, in particular its role in vitamin $D$ physiology and diseases that are marked by relative degrees of target tissue resistance to active vitamin $D$ metabolites.

\section{Conflict of Interest}

The authors have no financial relationships to disclose and no conflicts of interest.

\section{Acknowledgements}

Research reported in this publication was supported by the National Institute of Arthritis and Musculoskeletal and Skin Diseases of the National Institutes of Health under Award Number 5R01AR037399. The content is solely the responsibility of the authors and does not necessarily represent the official views of the National Institutes of Health. We thank the UCLA Vector Core (Emmanuelle Faure and Kip Hermann) for vector and viral preparations supported by JCCC/P30 CA016042 and CURE/P30 DK41301. We thank Duilio Casio for the computational facility at UCLA-DOE Institute of Genomics and Proteomics.

\section{References}

1 Carlberg C, Campbell MJ. Vitamin D receptor signaling mechanisms: integrated actions of a well-defined transcription factor. Steroids 2013; 78: 127-136.

2 Pike JW, Meyer MB. The vitamin D receptor: new paradigms for the regulation of gene expression by 1, 25-dihydroxyvitamin D3. Rheum Dis Clin North Am 2012; 38: 13-27.

3 Haussler MR, Whitfield GK, Kaneko I et al. Molecular mechanisms of vitamin d action. Calcif Tissue Int 2013; 92: 77-98.

4 Haussler MR, Haussler CA, Whitfield GK et al. The nuclear vitamin D receptor controls the expression of genes encoding factors which feed the "Fountain of Youth" to mediate healthful aging. J Steroid Biochem Mol Biol 2010; 121: 88-97.

5 Chen H, Hu B, Allegretto EA, Adams JS. The vitamin D response elementbinding protein. A novel dominant-negative regulator of vitamin Ddirected transactivation. J Biol Chem 2000; 275: 35557-35564.

6 Adams JS, Chen H, Chun RF et al. Novel regulators of vitamin D action and metabolism: lessons learned at the Los Angeles zoo. J Cell Biochem 2003; 88: 308-314.

7 Chen $\mathrm{H}$, Hewison M, Hu B, Adams JS. Heterogeneous nuclear ribonucleoprotein (hnRNP) binding to hormone response elements: a cause of vitamin D resistance. Proc Natl Acad Sci USA 2003; 100: 6109-6114.

8 Chen H, Hewison M, Adams JS. Functional characterization of heterogeneous nuclear ribonuclear protein $\mathrm{C} 1 / \mathrm{C} 2$ in vitamin $\mathrm{D}$ resistance: a novel response element-binding protein. J Biol Chem 2006; 281: 39114-39120.
9 Lisse TS, Hewison M, Adams JS. Hormone response element binding proteins: novel regulators of vitamin D and estrogen signaling. Steroids 2011; 76: 331-339.

10 Krecic AM, Swanson MS. hnRNP complexes: composition, structure, and function. Curr Opin Cell Biol 1999; 11: 363-371.

11 Carpenter B, MacKay C, Alnabulsi A et al. The roles of heterogeneous nuclear ribonucleoproteins in tumour development and progression. Biochim Biophys Acta 2006; 1765: 85-100.

12 Dreyfuss G. Structure and function of nuclear and cytoplasmic ribonucleoprotein particles. Annu Rev Cell Biol 1986; 2: 459-498.

13 Nakagawa TY, Swanson MS, Wold BJ, Dreyfuss G. Molecular cloning of cDNA for the nuclear ribonucleoprotein particle $C$ proteins: a conserved gene family. Proc Natl Acad Sci USA 1986; 83: 2007-2011.

14 Merrill BM, Barnett SF, LeStourgeon WM, Williams KR. Primary structure differences between proteins $\mathrm{C} 1$ and $\mathrm{C} 2$ of HeLa 40S nuclear ribonucleoprotein particles. Nucleic Acids Res 1989; 17: 8441-8449.

15 Mahajan MC, Narlikar GJ, Boyapaty G, Kingston RE, Weissman SM. Heterogeneous nuclear ribonucleoprotein $\mathrm{C} 1 / \mathrm{C} 2$, MeCP1, and SWI/ SNF form a chromatin remodeling complex at the beta-globin locus control region. Proc Natl Acad Sci USA 2005; 102: 15012-15017.

16 Irimura S, Kitamura K, Kato N et al. HnRNP C1/C2 may regulate exon 7 splicing in the spinal muscular atrophy gene SMN1. Kobe J Med Sci 2009; 54: E227-E236.

17 Izquierdo JM. Heterogeneous ribonucleoprotein $C$ displays a repressor activity mediated by T-cell intracellular antigen-1-related/like protein to modulate Fas exon 6 splicing through a mechanism involving Hu antigen R. Nucleic Acids Res 2010; 38: 8001-8014.

18 Shetty S. Regulation of urokinase receptor mRNA stability by hnRNP C in lung epithelial cells. Mol Cell Biochem 2005; 272: 107-118.

19 McCloskey A, Taniguchi I, Shinmyozu K, Ohno M. hnRNP C tetramer measures RNA length to classify RNA polymerase II transcripts for export. Science 2012; 335: 1643-1646.

20 Lisse TS, Liu T, Irmler M et al. Gene targeting by the vitamin D response element binding protein reveals a role for vitamin D in osteoblast mTOR signaling. Faseb J 2011; 25: 937-947.

21 Lisse TS, Hewison M. Vitamin D: a new player in the world of mTOR signaling. Cell Cycle 2011; 10: 1888-1889.

22 Lisse TS, Hewison M, Adams JS. Hormone response element binding proteins: Novel regulators of vitamin D and estrogen signaling. Steroids 2011; 76: 331-339.

23 Adams JS, Chen H, Chun R et al. Response element binding proteins and intracellular vitamin D binding proteins: novel regulators of vitamin D trafficking, action and metabolism. J Steroid Biochem Mol Biol 2004; 89/90: 461-465.

24 Chen H, Arbelle JE, Gacad MA, Allegretto EA, Adams JS. Vitamin D and gonadal steroid-resistant New World primate cells express an intracellular protein which competes with the estrogen receptor for binding to the estrogen response element. J Clin Invest 1997; 99: 669-675.

25 Soneoka Y, Cannon PM, Ramsdale EE et al. A transient three-plasmid expression system for the production of high titer retroviral vectors. Nucleic Acids Res 1995; 23: 628-633.

26 Chen C, Okayama H. High-efficiency transformation of mammalian cells by plasmid DNA. Mol Cell Biol 1987; 7: 2745-2752.

27 Whitson SR, LeStourgeon WM, Krezel AM. Solution structure of the symmetric coiled coil tetramer formed by the oligomerization domain of hnRNP C: implications for biological function. J Mol Biol 2005; 350: 319-337.

28 Phillips JC, Braun R, Wang W et al. Scalable molecular dynamics with NAMD. J Comput Chem 2005; 26: 1781-1802. 
29 Brooks BR, Brooks CL 3rd, Mackerell AD Jr et al. CHARMM: the biomolecular simulation program. J Comput Chem 2009; 30: 1545-1614.

30 Case DA, Cheatham TE 3rd, Darden T et al. The Amber biomolecular simulation programs. J Comput Chem 2005; 26: 1668-1688.

31 de Felipe P, Martin V, Cortes ML, Ryan M, Izquierdo M. Use of the 2A sequence from foot-and-mouth disease virus in the generation of retroviral vectors for gene therapy. Gene Ther 1999; 6: 198-208.

$32 \mathrm{Kim}$ JH, Lee SR, Li LH et al. High cleavage efficiency of a 2A peptide derived from porcine teschovirus-1 in human cell lines, zebrafish and mice. PLoS ONE 2011; 6: e18556.

33 Liu J, Zheng Q, Deng Y et al. A seven-helix coiled coil. Proc Natl Acad Sci USA 2006; 103: 15457-15462.

34 Burd CG, Dreyfuss G. RNA binding specificity of hnRNP A1: significance of hnRNP A1 high-affinity binding sites in pre-mRNA splicing. EMBO J 1994; 13: 1197-1204.

35 Swanson MS, Nakagawa TY, LeVan K, Dreyfuss G. Primary structure of human nuclear ribonucleoprotein particle $\mathrm{C}$ proteins: conservation of sequence and domain structures in heterogeneous nuclear RNA, mRNA, and pre-rRNA-binding proteins. Mol Cell Biol 1987; 7: 1731-1739.
36 McAfee JG, Shahied-Milam L, Soltaninassab SR, LeStourgeon WM. A major determinant of hnRNP C protein binding to RNA is a novel bZIP-like RNA binding domain. RNA 1996; 2: 1139-1152.

37 Shahied L, Braswell EH, LeStourgeon WM, Krezel AM. An antiparallel four-helix bundle orients the high-affinity RNA binding sites in hnRNP C: a mechanism for RNA chaperonin activity. J Mol Biol 2001; 305: 817-828.

38 Burd CG, Dreyfuss G. Conserved structures and diversity of functions of RNA-binding proteins. Science 1994; 265: 615-621.

39 Dreyfuss G, Matunis MJ, Pinol-Roma S, Burd CG. hnRNP proteins and the biogenesis of mRNA. Annu Rev Biochem 1993; 62: 289-321.

40 Takimoto $\mathrm{M}$, Tomonaga $\mathrm{T}$, Matunis $\mathrm{M}$ et al. Specific binding of heterogeneous ribonucleoprotein particle protein $\mathrm{K}$ to the human c-myc promoter, in vitro. J Biol Chem 1993; 268: 18249-18258.

This work is licensed under a Creative Commons AttributionNonCommercial-NoDerivs 3.0 Unported License. The images or other third party material in this article are included in the article's Creative Commons license, unless indicated otherwise in the credit line; if the material is not included under the Creative Commons license, users will need to obtain permission from the license holder to reproduce the material. To view a copy of this license, visit http:/ / creativecommons.org/licenses/by-nc-nd/3.0/

Supplementary Information for this article can be found on the Bone Research's website (http://www.nature.com/boneres). 